\title{
Diagnostic de la paralysie aiguë : application à l'épidémiologie des maladies virales de l'abeille en France en 1990
}

\author{
JP Faucon, C Vitu, P Russo, M Vignoni \\ Centre national d'études vétérinaires et alimentaires, laboratoire de pathologie des petits ruminants \\ et des abeilles, Route des Colles, Sophia Antipolis, 06410 Biot, France
}

(Reçu le 9 décembre 1991; accepté le 17 janvier 1992)

\begin{abstract}
Résumé - Varroa jacobsoni est le vecteur du virus de la paralysie aiguë. L'injection par l'acarien de ce virus dans l'hémolymphe entraîne des symptômes particuliers au niveau du couvain et des abeilles adultes. Ces symptômes peuvent être confondus avec ceux des loques, du couvain sacciforme ou de l'acariose. Dans un premier temps, le diagnostic du virus de la paralysie aiguë par immunodiffusion en gélose a été mis au point. Puis durant l'année 1990, le diagnostic des maladies virales reconnues comme les plus importantes a été appliqué aux prélèvements reçus au laboratoire ou effectués au cours des visites de rucher, cela afin de préciser la fréquence et de connaître les possibles associations de ces maladies entres elles ou avec la varroase. Le virus de la paralysie aiguë a été retrouvé dans $9,8 \%$ des cas, toujours associé à la varroase. Une forte progression de la loque américaine et à un moindre degré de la loque européenne et du couvain sacciforme a été constatée. En ce qui concerne les maladies des abeilles adultes, la maladie noire est en progression.
\end{abstract}

virus de la paralysie aiguë / maladle virale / épidémiologie / varroatose

\section{INTRODUCTION}

La période (année 1990) qui a suivi les années de forte infestation en varroas, durant lesquelles les traitements étaient insuffisamment efficaces, voit l'apparition ou la recrudescence de differentes affections virales : paralysie aiguë (Acute bee paralysis virus - APV), paralysie chronique ou maladie noire (Chronic bee paralysis virus - CPV), couvain sacciforme (Sacbrood bee virus - SBV).
Le virus de la paralysie aiguë mis en évidence dans les années 1960 (Bailey et al, 1963; Giauffret et al, 1969) n'avait, avant l'apparition de la varroase, aucun rôle pathogène. II n'était détecté que par des tests en laboratoire et n'était à l'origine d'aucune mortalité dans la nature. Avec l'apparition de la varroase, la mise en évidence du virus de la paralysie aiguë s'est multipliée, avec constatation de signes cliniques d'autant plus répandus que l'infestation par Varroa jacobsoni gagnait en importance (Ball et Allen, 1988). Mais 
du fait de son inoculation par l'acarien Varroa jacobsoni, une augmentation importante de son développement et à fortiori de son pouvoir pathogène lié au seuil d'infestation croissant des colonies en varroa a été enregistré (Ball et Allen, 1988). Les symptômes que ce virus induit chez le couvain et chez les abeilles adultes peuvent être confondus avec ceux de la loque américaine, de la loque européenne, du couvain sacciforme et, dans une moindre mesure, avec ceux de l'acariose (Acarapis woodi). C'est pourquoi nous avons entrepris de mettre au point son diagnostic. Nous avons choisi l'immunodiffusion en gélose (IDG) car cette technique robuste et fiable est une méthode de référence, fréquente en pathologie animale, facile à mettre en œuvre. L'utilisation de I'ELISA a été envisagée, mais cette technique beaucoup plus sensible est surtout adaptée à la détection du virus, indépendamment de toute expression clinique (Allen et al, 1986) ce qui n'était pas notre objectif. Cela a permis de préciser les cas et les associations où le virus de la paralysie aiguë était présent, de dégager la part attribuée à la pathologie classique (loque américaine, loque européenne) et donc, de pouvoir orienter les apiculteurs confrontés à une symptomatologie complexe vers un mode de traitement approprié.

\section{MATÉRIEL ET MÉTHODES}

Les diagnostics effectués durant l'année 1990 ont porté sur 61 échantillons de couvain et 17 échantillons d'abeilles adultes.

\section{Diagnostic du couvain sacciforme}

La recherche du SBV a été effectuée par immunodiffusion (Giauffret, 1974).

\section{Diagnostic de la paralysie chronique}

La recherche de CPV (Giauffret et Lambert, 1972) a été effectuée par reproduction expérimentale de la maladie et confirmation de la présence de particules virales au microscope électronique.

\section{Détermination de l'infestation en varroas}

Le seuil d'infestation en Varroa a été déterminé par l'examen de 100 alvéoles operculées.

\section{Diagnostic du virus de la paralysie aiguë}

La mise au point de ce diagnostic dérive de la technique utilisée pour le SBV.

Le virus de la paralysie aiguë a été isolé à partir de couvains fortement infestés par Varroa, présentant des symptômes de loques mais sans qu'un examen direct ne révèle la présence d'agents microbiens.

Dix larves malades ont été broyées au mortier de Griffith dans $5 \mathrm{ml}$ d'eau physiologique (solution de $\mathrm{NaCl}$ à $8,5 \%$ ). Après élimination des possibles contaminations bactériennes par centrifugation ( $30 \mathrm{~min}$ à $3800 \mathrm{~g}$ ) et dilution dans de l'eau physiologique jusqu'à $10^{-6}$, une injection intrathoracique de $2 \mu \mathrm{l}$ de cette dilution à des abeilles saines a été réalisée. La mortalité dans des cagettes en altuglas contenant 30 abeilles inoculées maintenues à $35^{\circ} \mathrm{C}$ et $50 \%$ d'hygrométrie a été quasi totale en $3 \mathrm{j}$ maximum. Pour des témoins traités de la même manière, par injection de $2 \mu \mathrm{l}$ d'eau physiologique, elle n'a pas excédé $6,5 \%$ pour cette même période.

Les particules virales ont été identifiées par microscopie électronique sur des broyats de ces abeilles mortes. Le virus de la paralysie aiguë est de forme parasphérique et mesure $30 \mathrm{~nm}$. L'identification a été confirmée par BV Ball (Rothamsted - Experimental station).

Plusieurs inoculations ont ensuite été réalisées avec de nouveaux extraits préparés à partir de ces abeilles mortes afin d'obtenir suffisam- 
ment de matériel pour l'isolement et la purification du virus.

\section{Purification du virus}

\section{Broyat initial}

Deux cent quatre vingt sept abeilles adultes, mortes de maladie expérimentale, ont été broyées à l'ultra-Turrax dans $200 \mathrm{ml}$ de PBS additionnés d'azide de sodium à $0,02 \%$ (PBSa). Après filtration sur gaze, l'extrait a été clarifié à $8000 \mathrm{tr} / \mathrm{min}-10000 \mathrm{gr} r_{\text {max }}$, le surnageant récupéré.

\section{Centrifugation sur gradient}

L'isolement des particules virales a été réalisé par ultracentrifugation sur gradient de saccharose en rotor zonal (rotor titane B XIV, $650 \mathrm{ml}$ ) sur centrifugeuse MSE superspeed 75 , selon une technique antérieurement éprouvée (Giauffret et al, 1973; Vitu et al, 1978).

Le rotor a été chargé par la périphérie avec, successivement :

- un overlay : $60 \mathrm{ml}$ de saccharose à $7 \% \mathrm{pp}$;

- l'échantillon : $180 \mathrm{ml}$ de broyat clarifié, amené à $9 \%$ de saccharose $\mathrm{pp}$;

- le gradient préformé sur mélangeur avec 200 $\mathrm{ml}$ de saccharose à $10 \% \mathrm{pp}$ et $200 \mathrm{ml}$ à $55 \%$ pp;

- un coussin de saccharose à $55 \% \mathrm{pp}$;

Après $4 \mathrm{~h}$ de rotation à $40000 \mathrm{tr} / \mathrm{min}$ à $120000 \mathrm{gr}_{\max }$, le contenu du rotor a été poussé par introduction de saccharose à $58 \% \mathrm{pp}$. Des fractions de $10 \mathrm{ml}$ ont été récupérées sur collecteur ISCO 1220 avec enregistrement simultané de la densité optique à $260 \mathrm{~nm}$ (moniteur ISCO UA5). La courbe du gradient a été obtenue par réfractométrie des différentes fractions, les résultats de la séparation sont résumés dans la figure 1.

D.O. $260 \mathrm{~nm}$

$\%$ saccharose

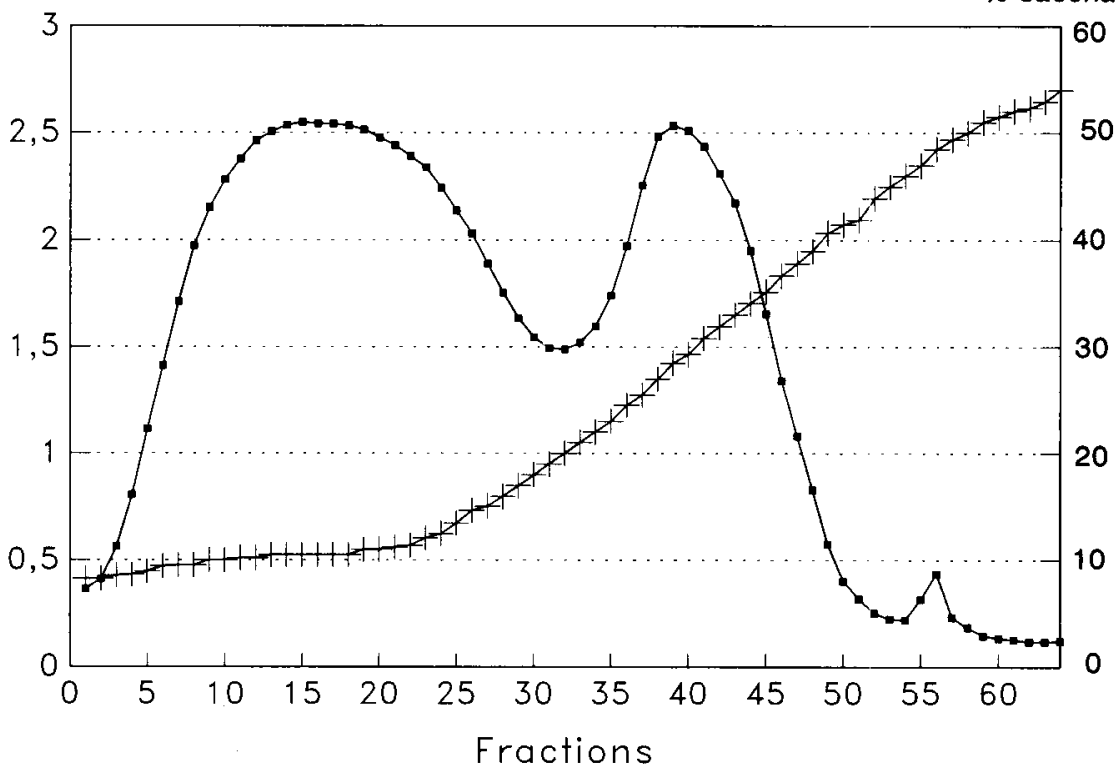

- Série 1 - Série 2

Fig 1. Profil d'élution du virus APV purifié en rotor zonal. Série 1 = étude de l'absorbance (DO) à 260 $\mathrm{nm}$ sur $0,1 \mathrm{~cm}$ montrant le pic viral (F56) bien détaché du matériel cellulaire, localisé à $48 \% \mathrm{pp}$ de saccharose; série 2 = courbe du gradient de saccharose. 


\section{Contrôle des fractions}

La richesse et la pureté virale des fractions du dernier pic (autour de la fraction 56) ont été contrôlées par microscopie électronique (coloration négative à l'acide phosphotungstique (fig 2), et par leur spectre d'absorption entre 300 et $200 \mathrm{~nm}$ (spectrophotomètre Philips PU 8800), indiquant un maximum d'absorption à $255 \mathrm{~nm}$ caractéristique des acides nucléiques.

\section{Préparation de l'immunsérum et réaction IDG}

Après dilution dans un volume égal de PBSa, les fractions 54 à 60 ont été récupérées en culot par centrifugation de $1 \mathrm{~h}$ à $40000 \mathrm{tr} / \mathrm{min}-$ $180000 \mathrm{~g} r_{\max }$ sur centrifugeuse Beckman L8$60 \mathrm{M}$. Les culots ont été repris par $2,5 \mathrm{ml}$ de PBS pour constituer l'inoculum.

Un lapin a été immunisé par 5 inoculations successives réalisées à $8 \mathrm{j}$ d'intervalle de $0,5 \mathrm{ml}$ d'inoculum viral émulsionné dans un égal volume d'adjuvant complet de Freund et administré par voie intradermique en points multiples. Le sérum a été prélevé $6 \mathrm{j}$ après la dernière immu- nisation, son pouvoir précipitant a été contrôlé dans la réaction d'IDG vis-à-vis de l'inoculum et d'extraits d'abeilles infectées.

L'IDG a été réalisée sur gélose à $1 \%$ (Indubiose $\mathrm{A} 37 \mathrm{IBF}$ ) en tampon véronal-soude $\mathrm{pH}$ 8,142 , coulée sur lame porte objet $25 \times 75 \mathrm{~mm}$ : 6 puits de $3 \mathrm{~mm}$ de diamètre $(20 \mu /$ d'échantillon) sont disposés en couronne autour du puits central (distance des centres des puits au centre du puits sérum : $8,5 \mathrm{~mm}$ ). Un témoin positif (broyat d'abeilles APV) et un témoin négatif (broyat de larves saines) sont disposés sur chaque couronne. Les lignes de précipitation sont visibles après une nuit en chambre humide : la présence de virus dans les échantillons testés fait apparaître un arc de précipitation spécifique, en continuité avec la ligne du témoin positif APV, et situé tout près des puits périphériques.

\section{RÉSULTATS}

Le diagnostic par IDG a été testé sur abeilles adultes et sur couvains malades afin de définir la sensibilité et la spécificité de la réaction. Si une ligne très nette est obtenue avec un broyat de 4 abeilles ino-

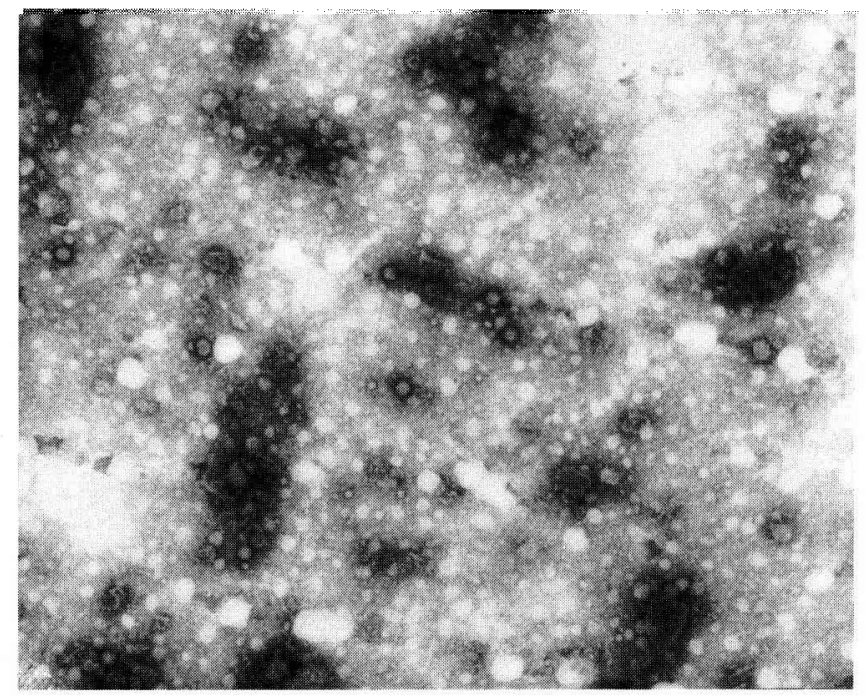

Fig 2. Particules du virus de la paralysie aiguë. Contraste négatif ( $x 60000)$. 
culées dans $1 \mathrm{ml}$ d'eau physiologique, la réaction est cependant optimale en utilisant 4 abeilles entières ou 4 larves atteintes et en les broyant dans un mortier de Griffith avec 5 gouttes $(250 \mu l)$ d'eau physiologique.

Aucune réaction n'a été enregistrée visà-vis de SBV ou de CPV (fig 3). De même, le sérum anti-SBV ne réagit pas avec un broyat de APV.
Dans les examens d'abeilles adultes (tableau II), il y a augmentation de la paralysie chronique $(53 \%)$. La mise en évidence de CPV en microscopie électronique (fig 4) a confirmé le diagnostic par reproduction expérimentale de la maladie. L'acariose est aussi fréquemment rencontrée. L'APV n'a par contre jamais été mis en évidence.

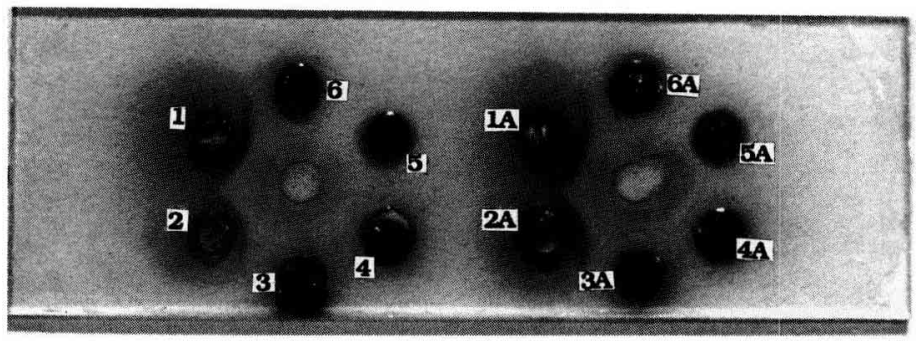

Fig 3. Immunodiffusion en gélose. Au centre sérum de lapin anti APV; $n^{\circ} 1$ et $1 A$ témoin APV; $n^{\circ} 2$ et $2 A$ échantillons d'abeilles malades (APV); $n^{\circ} 3$ et $3 A$ larves saines; $n^{\circ} 4$ et $4 A$ SBV; $n^{\circ} 5$ et $5 A$ CPV; $n^{\circ} 6$ et $6 A$ échantilion négatif en APV.

Des lignes de précipitation mineures sont parfois visibles entre le puits central et l'arc de précipitation virale : il s'agit d'une faible réactivité de l'immunsérum visà-vis des antigènes de l'abeille saine, le sérum n'ayant pas été épuisé contre les antigènes de l'abeille saine afin de ne pas diminuer sa réactivité anti APV. Toutefois, il ne peut $y$ avoir de confusion entre l'arc "virus" et la ligne interne "protéine abeille" montrée par le témoin “larve saine".

Dans les examens de couvain (tableau I), nous constatons par rapport à nos observations personnelles des années antérieures une nette augmentation de la loque américaine $(47,5 \%)$ ainsi que de la loque européenne $(32,8 \%)$ et une faible augmentation du couvain sacciforme $(9,8 \%)$ et de l'APV $(9,8 \%)$.
Tableau I. État de la pathologie au niveau du couvain en 1990. $L A=$ Loque américaine; $L E=$ Loque européenne; SBV = Couvain sacciforme; APV = virus de la paralysie aiguë; IF + = Infestation en varroas positive; MND = Mortalité non déterminée. Pourcentage $=$ Pourcentage par rapport au nombre de prélèvements sur 61 couvains.

\begin{tabular}{lcc}
\hline Pathologies & Nombre & Pourcentage \\
\hline LA & 29 & 47,5 \\
LE & 20 & 32,8 \\
LA + LE & 1 & - \\
SBV & 6 & 9,8 \\
LE + SBV & 2 & - \\
APV & 6 & 9,8 \\
APV + LE & 2 & - \\
APV + LA & 0 & - \\
APV + SBV & 0 & - \\
IF + & 6 & - \\
MND & 0 & - \\
\hline
\end{tabular}


Tableau II. État de la pathologie au niveau des abeilles adultes en 1990. CPV $=$ Paralysie chronique; $A P V=$ Paralysie aiguë; $A=$ Acariose (Acarapis woodi); $\mathrm{MND}=$ Mortalité non déterminée. Pourcentage $=$ Pourcentage par rapport au nombre de prélèvements sur 17 échantillons d'abeilles adultes.

\begin{tabular}{lcc}
\hline Pathologies & Nombre & Pourcentage \\
\hline CPV & 9 & 53 \\
APV & 0 & - \\
CPV + APV & 0 & - \\
A & 3 & 17,6 \\
MND & 5 & 29,4 \\
\hline
\end{tabular}

en les distinguant des autres pathologies possibles, ce qui était notre objectif.

La mise au point du diagnostic de l'APV par immunodiffusion a permis durant l'année 1990 de préciser quelle part pouvait être attribuée à ce virus dans les problèmes de pathologie rencontrés.

Le virus de la paralysie aiguë a été retrouvé dans $9,8 \%$ des cas, au niveau du couvain seulement et chaque fois que l'infestation par varroa était positive. Nous rejoignons en cela les conclusions de Ball (1985).

Le virus de I'APV est à prendre en compte dans l'étiologie complexe de la

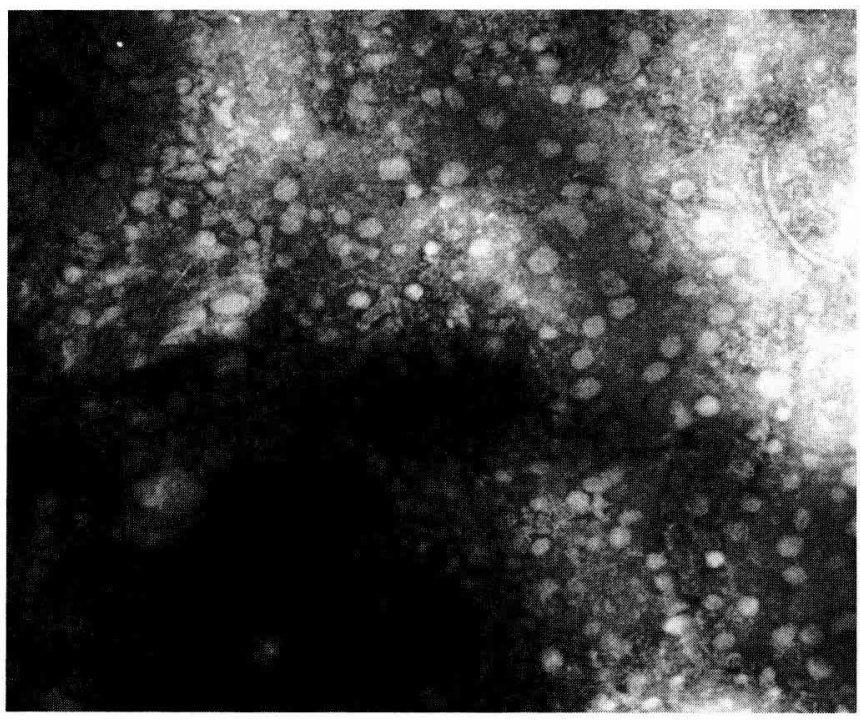

Fig 4. Particules du virus de la paralysie chronique. Contraste négatif ( $x 80000$ ).

\section{DISCUSSION ET CONCLUSION}

Le diagnostic de l'APV par IDG est apparu spécifique. Sa sensibilité est suffisante pour détecter les cas de paralysie aiguë, loque européenne, comme le virus du couvain sacciforme d'ailleurs.

Considérant les autres pathologies, nous constatons une forte progression de la loque américaine et, à un degré moin- 
dre, de la loque européenne et du couvain sacciforme. Le nombre des échantillons reçus a doublé entre 1989 et 1990. L'explication du développement de ces affections n'est pas claire. L'histoire apicole a déjà montré de tels phénomènes. Ils peuvent être attribués à différents facteurs parmi lesquels : l'apport de toxiques de différentes origines affaiblissant les colonies (observations personnelles), l'abandon de la prophylaxie vis-à-vis de ces maladies au profit de la lutte contre la varroase, les conditions météorologiques défavorables (sécheresse intense induisant des carences alimentaires), le confinement des abeilles.

En ce qui concerne les abeilles adultes, aucun cas de paralysie aiguë n'a été mis en évidence.

On notera que l'acariose, malgré l'utilisation des produits acaricides pour lutter contre la varroase, est toujours présente.

La maladie noire, par contre, a été en extension durant toute l'année. Elle peut expliquer en partie les dépopulations et l'affaiblissement des colonies constatés en août dans certaines régions apicoles. $\mathrm{Ce}$ pendant, l'association avec des conditions climatiques défavorables qui limitent le butinage et favorisent la transmission des agents pathogènes, la surpopulation en colonies des régions viennent se surajouter (Bailey et al, 1983). Les pesticides retrouvés à des doses sublétales semblent intervenir aussi comme cause favorisante (observations et expériences personnelles). Des expérimentations sont en cours pour préciser exactement l'importance de ces différents facteurs sur le développement du CPV.

Summary - Diagnosis of acute paralysis: application to epidemic honeybee diseases in France during 1990. Varroa jacobsoni is the vector of acute paralysis virus (APV). Its injection into the hemolymph by the acarian mite induces particular symptoms in brood and bees. These symptoms can be mistaken for those of foulbrood, sacbrood or acarian disease. At first, the diagnosis of APV was determined by gel immunodiffusion (IDG): the virus was isolated from larvae infected by APV and inoculated into healthy bees maintained at $35{ }^{\circ} \mathrm{C}$ and with $50 \%$ of hygrometry. Two or three days later, the virus was isolated from dead bees and purified, and then an immune serum prepared in rabbit and used for IDG diagnosis. In 1990, the diagnosis of APV, chronic paralysis (CPV) and sacbrood (SVB) was made in all samples sent to the laboratory. In 78 samples, APV was found only 6 times, twice associated with European foulbrood. On the other hand, an increase in American foulbrood, European foulbrood and sacbrood was found. The decrease in prophylaxis of brood diseases to the exclusive detriment of varroatosis treatment, and the widespread use of pesticides against Varroa jacobsoni are probable explanations for this increase.

At the foraging bee level, the chronic paralysis virus diagnosed in a large number of hives partly explains the depopulation. The synergistic effect between pesticides and meteorological conditions should also be taken into consideration.

acute paralysis / viral disease / epidemiology / varroatosis

\section{Zusammenfassung - Diagnose der akuten Paralyse: Beziehungen zu epide- mischen Bienenkrankheiten in Frank- reich im Jahre 1990. Varroa ist der Über- träger des akuten Paralyse-Virus (APV). Seine Injektion in das Bienenblut durch die Milbe verursacht bestimmte Symptome bei Brut und Arbeitsbienen. Diese Symptome}


können mit denen von Faulbrut, Sackbrut oder Tracheenmilben verwechselt werden. Zunächst wurde die Diagnose des APV mittels der Gel-Immundiffusion (IDG) durchgeführt. $\mathrm{Zu}$ diesem Zweck wurde das Virus zunächst von mit APV infizierten Larven isoliert und in gesunde Bienen injiziert, die bei $15^{\circ} \mathrm{C}$ und $50 \%$ Feuchte im Brutschrank gehalten wurden. Zwei oder drei Tage später wurde das Virus von toten Bienen isoliert und gereinigt; anschließend wurde ein Kaninchen-Immunserum erzeugt, das zur IDG-Diagnose benutzt werden konnte. Im Jahre 1990 wurden bei allen eingesandten Proben diagnostische Untersuchungen auf APV, chronische Paralyse (Schwarzsucht, CPV) und Sackbrut (SVB) durchgeführt. Bei 78 Proben wurde APV nur sechs mal gefunden, davon zweimal zusammen mit Europaïscher Faulbrut. Andererseits nahmen Amerikanische Faulbrut, Europäische Faulbrut und Sackbrut deutlich zu. Abnahme der Prophylaxe gegen Brutkrankheiten zugunsten der ausschlieBlichen Behandlung der Varroatose und die umfangreiche Verwendung von Pestiziden zur $\mathrm{Be}$ handlung von Varroa jacobsoni sind die wahrscheinlichen Ursachen dieser Entwicklung. Der Verlust an Trachtbienen wird zumindest teilweise durch die Diagnose des Virus der Chronischen Paralyse erklärt, die in zahlreichen Völkern gefunden wurde. Man muß aber auch ein Zusammenwirken von Pestiziden mit den meteorologischen Bedingungen in Betracht ziehen.

\section{akute Paralyse / Viruskrankheit / Epide- miologie / Varroatose}

\section{REFERENCES}

Allen MF, Ball BV, White RF, Antoniw JF (1986) The detection of acute paralysis virus in Varroa jacobsoni by the use of a simple direct ELISA. J Apic Res 25, 100-105

Bailey L, Gibbs AJ, Woods RD (1963) Two viruses from adult honeybee. Virology 21, 390395

Bailey L, Ball BV, Perry JN (1983) Honeybee paralysis: its natural spread and its diminished incidence in England and Wales. J Apic Res 22, 191-195

Ball BV (1985) Acute paralysis virus isolates from honeybee colonies infested with Varroa jacobsoni. J Apic Res 24, 115-119

Ball BV, Allen MF (1988) The prevalence of pathogens in honeybee (Apis mellifera) colonies infested with the parasitic mite Varroa jacobsoni. Ann Appl Biol 113, 237-244

Giauffret A, Duthoit JL, Poutiers F, Tostain Caucat MJ (1969) Étude de différentes souches virales isolées chez l'abeille adulte (Apis mellifera). Bull Apic 12, 13-22

Giauffret A, Lambert M (1972) Le diagnostic de la Maladie noire de l'abeille. Rec Med Vet 148, 869-877

Giauffret A, Vitu C, Lambert M (1973) Purification de différents virus libres d'invertébrés en rotor zonal. European Symposium of zonal centrifugation in density gradient. Spectra $20004,245-252$

Giauffret A (1974) Couvain sacciforme de l'abeille (Sacbrood). In: Diagnostic séroimmunologique des viroses humaines et animales (Bricout F, Joubert L, Huraux JM, eds) Maloine, Paris

Vitu C, Russo P, Lambert M, Giauffret A (1978) Purification de Chlamydia psittaci par centrifugation en rotor zonal. Rev Med Vét 129, 1187-1198 